\title{
A Mathematical Model of Dracunculiasis Epidemic and Eradication
}

\author{
Matthew O. Adewole ${ }^{1}$, Akindele A. Onifade ${ }^{2}$ \\ ${ }^{1,2}$ Department of Mathematics, University of Ibadan, Ibadan, Nigeria.
}

\begin{abstract}
We present a mathematical model for the spread of dracunculiasis with focus on three populations; human, vector and parasite. The reproductive number is obtained from next generation matrix and the stability analyses of disease-free and endemic equilibria are conducted. Simulation of the model is presented by solving the systems of the differential equations to explore the behaviour of the model using maple 14. The paper also analyzes key parameters to determine the effective intervention. The result of this paper shows that reducing the parasite birth rate is more effective than water treatment.
\end{abstract}

Keywords: Copepod, dracunculiasis, reproductive number, stability.

\section{Introduction}

Dracunculiasis (Dracontiasis), more generally called Guinea Worm Disease (GWD), is a serious problem in various countries in Africa. It is a parasitic infection acquired by drinking water from ponds contaminated by cyclopoid copepods infected with third stage larvae of the parasite Dracunculusmedinensis. Worm emergence is through the skin after a year of entering the infected person and this is usally associated with secondary bacterial infection. It has been called a neglected disease of neglected people since it strikes remote farming populations who have been passed over by national development efforts [1]. Intervention and prevention techniques have been implemented in endemic areas to significantly reduce outbreaks. Dracunculiasis is a disease of poor rural communities where the population often has to obtain drinking water from ponds infested with water fleas called Cyclops. Guinea worm eggs are not directly infective to humans. They can remain active in water for about three days and die unless they are swallowed by a cyclops. Inside the cyclops, the guinea-worm larvae develop over a period of about two weeks into a larval stage that is infective to humans [2]. The Cyclops become inactive after infection and die early [3].

There are no symptoms during the year long incubation period. High susceptibility to tetanus through ulcers caused by the emerging worms in addition to habitual abortion in some pregnant women [4] has been associated with guinea-worm diseases. When the adult worms are immersed in water, they release hundreds of thousands of mature larvae to begin the cycle anew. Each infection lasts only one year, but more than one guinea worm may emerge simultaneously or sequentially over the course of weeks, depending on the number and intensity of infection the preceding year. Humans do not develop immunity, and there is no cure or vaccine for the infection. However, the worm can be removed by physically pulling the worm out which may take up to two months to complete as worm can grow up to a meter in length and only $1-2 \mathrm{~cm}$ can be removed per day [5]. Dracunculiasis can only be prevented by teaching persons to always filter drinking water from unsafe sources through a fine cloth and to avoid entering such sources when they have a worm emerging or about to emerge from their bodies, by treating contaminated water with ABATE larvicide (temephos; BASF, Mount Olive, NJ) or by providing safe drinking water from underground sources.

Considerable progress has been made since 1986 in reducing the annual numberof reported dracunculiasis cases. The 1991 world health assembly (WHA) goal to eradicate dracunculiasis globally by 1995 was not achieved because of the limited funding available from international organization for support of technical and financial assistance to countries with endemic disease, and the limited time (4years) to meet the WHA goal [6]. In 2004, WHA established a new target date of 2009 for global eradication [7] despite considerable progress, that target also was not met, nevertheless, progress towards eradication continues. The number of cases of dracunculiasis worldwide reported by disease endemic countries to WHO and partner organizations decreased by 41 percent, from 1,797 cases in 2010 to 1,058 in 2011. As of June 2012, dracunculiasis remained endemic in four countries (Chad, Ethiopia, Mali and South Sudan [7]. The 395 cases reported and 219 villages reporting cases globally during January to June 2012 represent reductions of $51 \%$ and $39 \%$, respectively, from the 807 cases reported and 358 villages that reported cases during January to June 2011. Of the 395 cases reported during January to June 22012, 99\% were from South Sudan [7].

Few researchers have contributed towards the mathematical study of the eradication of dracunculiasis (guinea worm) diseases.Adetunde [8] investigated the current pattern of dracunculiasis disease in the Northern region of Ghana. He analysed the data from the region and wrote a time series model for the purpose of prediction. From his analysis it was observed that the number of Guinean infection cases reduce with time and 
concluded that if the trend continues then there is likelihood that the guinea worm disease will be completely eradicated.

Recently, KathryhLink [9] in her M.Scthesis, highlighted compartmental modelling of the biological description of the disease. The model provides the basis for examining the guinea worm diseases hostmicroparasite interaction. An algebraic solution to disease-free equilibrium was found and a numerical stability analysis of the solution was conducted. Using next generation matrix, she determined the reproductive number $\mathrm{R}_{0}$, which enabled her to discover that the disease-free equilibrium is stable provided the people's visitation rate to the river/water body is reduced.

Robert J. Smith et al., [5] developed a mathematical model of guinea worm disease. Impulsive differential equations were used to evaluate the effectiveness of chlorination. Latin Hypercube sampling was used to determine the practical effectiveness of three control parameters (education, filtration and chlorination). Despite the theoretical potential of chlorination to complete the eradication of the disease, education is far more effective.

In this paper we formulate a mathematical model to represent the spread of guinea worm disease among three different populations. We analyse the model for two intervention parameters, $\xi_{\mathrm{L}}$ (water treatment)and $\lambda_{0}$ (education) to check the more effective intervention.

The rest of this paper is organised as follows. Section 2 is devoted to model formulation. In section 3, we analyse the model and state the conditions that guarantee the stability of the disease-free and endemic equilibria, then the model is solved numerically. Conclusion is made in Section 4.

\section{Model Formulation}

Three different populations are considered in this paper, human, parasite and vector populations. The human population is divided into three compartments (or states) containing susceptible human $\mathrm{S}_{\mathrm{H}}$, exposed human $\mathrm{E}_{\mathrm{H}}$ and infected human $\mathrm{I}_{\mathrm{H}}\left(N_{H}=S_{H}+E_{H}+I_{H}\right)$. When a man ingests an infected copepod, he becomes exposed and later (after about one year when the guinea worm larva has fully grown) becomes infected.

In this model we assume that more than one guinea worm can emerge simultaneously or sequentially over the course of the weeks, depending on the number and the intensity of infection the preceding year.

No human death occurs due to guinea worm disease. The susceptible human state $S_{H}$ gains individuals through birth $\eta_{H} N_{H}\left(1-\frac{N_{H}}{K_{H}}\right)$, and recovery from infection $\kappa I_{H}$. A loss of individual is as a result of death $\xi_{H} S_{H}(1-$ $\left.\frac{N_{H}}{K_{H}}\right)$ and infection $\varepsilon_{C} \beta\left(\frac{I_{C}}{\Phi_{c}}\right) S_{H}$. The exposed human state $\mathrm{E}_{\mathrm{H}}$ gains individuals through infection $\varepsilon_{C} \beta\left(\frac{I_{C}}{\Phi_{c}}\right) S_{H}$ and loses individuals when they become infected $\alpha E_{H}$ and to natural death $\xi_{H} I_{H}\left(1-\frac{N_{H}}{K_{H}}\right)$. The infected human $I_{H}$ gains individuals when exposed individuals become infected and loses individuals when they die $\xi_{H} I_{H}\left(1-\frac{N_{H}}{K_{H}}\right)$ or recover $\kappa I_{H}$.

The susceptible copepod state $S_{C}$ gains more individuals only through birth $\eta_{C} S_{H}\left(1-\frac{N_{C}}{K_{C}}\right)$. The population loses copepods through natural death $\xi_{C} S_{C}\left(1-\frac{N_{C}}{K_{C}}\right)$, consumption by human $\beta\left(\frac{S_{C}}{\Phi_{C}}\right) N_{H}$, and to infection by guinea worm larvae, $\varepsilon_{L} \gamma\left(\frac{L}{\Phi_{\mathrm{L}}}\right) S_{C}$, $\left(N_{C}=S_{C}+I_{C}\right)$. The infected copepod state $I_{C}$ loses individual through death $\xi_{C}^{\prime} I_{C}\left(1-\frac{N_{C}}{K_{C}}\right)$ and consumption by human $\beta\left(\frac{I_{C}}{\Phi_{C}}\right) N_{H}$. Copepod can never recover from infection. In this paper it is assumed that the amount of copepods consumed per time by maximum population of human is less than the saturation population of copepods per time $\left(\right.$ i. e. $\left.0<\beta K_{H}<\Phi_{C}\left(\eta_{C}-\xi_{C}^{\prime}\right)\right)$.

The guinea worm population is represented by both eggs and larvae. The egg state $E$ gains more individual by the release of eggs from adult worms existing in human host $\lambda_{0} \lambda_{1} I_{H}$. The population loses eggs through natural death $\xi_{E} E$ and hatching $\omega E$ into the larvae. It is assumed that $\omega>\xi_{E}$. The larvae state $L$ gains more larvae by hatching of eggs $\omega \sigma E$. Losses occur due to natural death $\xi_{L} L$ and consumption of larvae by copepod $\gamma\left(\frac{L}{\Phi_{L}}\right) N_{C}$.

Thus the model is

$$
\begin{aligned}
& \quad \frac{d S_{H}}{d t}=\left(\eta_{H} N_{H}-\xi_{H} S_{H}\right)\left(1-\frac{N_{H}}{K_{H}}\right)+\kappa I_{H}-\varepsilon_{C} \beta\left(\frac{I_{C}}{\Phi_{C}}\right) S_{H} \\
& \frac{d E_{H}}{d t}=\varepsilon_{C} \beta\left(\frac{I_{C}}{\Phi_{C}}\right) S_{H}-\alpha E_{H}-\xi_{H} E_{H}\left(1-\frac{N_{H}}{K_{H}}\right) \\
& \frac{d I_{H}}{d t}=\alpha E_{H}-\kappa I_{H}-\xi_{H} I_{H}\left(1-\frac{N_{H}}{K_{H}}\right) \\
& \frac{d E}{d t}=\lambda_{0} \lambda_{1} I_{H}-\left(\omega+\xi_{E}\right) E
\end{aligned}
$$




$$
\begin{aligned}
& \frac{d L}{d t}=\omega \sigma E-\xi_{L} L-\gamma\left(\frac{L}{\Phi_{\mathrm{L}}}\right) N_{C} \\
& \quad \frac{d S_{C}}{d t}=\left(\eta_{C} N_{C}-\xi_{C} S_{C}\right)\left(1-\frac{N_{C}}{K_{C}}\right)-\varepsilon_{L} \gamma\left(\frac{L}{\Phi_{\mathrm{L}}}\right) S_{C}-\beta\left(\frac{S_{C}}{\Phi_{C}}\right) N_{H} \\
& \frac{d I_{C}}{d t}=\xi_{L} \gamma\left(\frac{L}{\Phi_{\mathrm{L}}}\right) S_{C}-\beta\left(\frac{I_{C}}{\Phi_{C}}\right) N_{H}-\xi_{C}^{\prime} I_{C}\left(1-\frac{N_{C}}{K_{C}}\right)
\end{aligned}
$$

Since the model monitors changes in the human, cyclops and parasite populations, the variables and the parameters are assumed to be non-negative for all $t \geq 0$. Therefore $(2.1)-(2.7)$ will be analysed is a suitable feasible region $\mathfrak{R}$ of biological interest. We have the following lemma on the region system $(2.1)-(2.7)$ are resisted to.

Lemma 2.1: The Feasible region $\mathfrak{R}$ defined by

$$
\begin{aligned}
\Re= & \left\{\left(S_{H}(t), E_{H}(t), I_{H}(t), E(t), L(t), S_{C}(t), I_{C}(t)\right) \in R_{+}^{7}: N_{H}(0) \leq N_{H}(t) \leq K_{H},\right. \\
& \left.0 \leq N_{H}(t)<K_{C}\right\}
\end{aligned}
$$

with initial conditions

$S_{H}(0) \geq 0, E_{H}(0) \geq 0, I_{H}(0) \geq 0, E(0) \geq 0, L(0) \geq 0, S_{C}(0) \geq 0, I_{C}(0) \geq 0$ is positive invariant for system (2.1)-(2.7).

Proof: Adding (2.1) - (2.3) we obtain

$$
\frac{d N_{H}}{d t}=\left(1-\frac{N_{H}(t)}{K_{H}}\right)\left(\eta_{H}-\xi_{H}\right) N_{H}(t)
$$

The assumption that $N_{H}(0) \leq K_{H}$ implies

$$
N_{H}(t)=\left\{\begin{array}{c}
\frac{K_{H} N_{H(0)}}{\left(K_{H}-N_{H(0)}\right) \exp \left(-\left(\eta_{H}-\xi_{H}\right) t\right)+N_{H}(0)} \text { if } N_{H}(0)<K_{H} \\
K_{H} \text { if } N_{H}(0)=K_{H}
\end{array}\right\} \leq K_{H}
$$

Using the facts that $N_{H}(t) \leq K_{H}$ and $\eta_{H} \geq \xi_{H}$ in (2.8) we have

thus, $N_{H}(0) \leq N_{H}(t) \leq K_{H}$.

$$
\frac{d N_{H}}{d t} \geq 0 \Rightarrow N_{H}(t) \geq N_{H}(0)
$$

Similarly, adding (2.6) and (2.7) we obtain

Since $\xi_{C}^{\prime}>\xi_{C}$ and $\beta\left(\frac{N_{C}}{\Phi_{C}}\right) N_{H} \geq 0$ we have

$$
\frac{d N_{C}}{d t}=\left(1-\frac{N_{C}}{K_{C}}\right)\left(\eta_{C} N_{C}-\xi_{C} S_{C}-\xi_{C}^{\prime} I_{C}\right)-\beta\left(\frac{N_{C}}{\Phi_{C}}\right) N_{H}
$$

which follows that

$$
\frac{d N_{C}}{d t}<\left(1-\frac{N_{C}(t)}{K_{C}}\right)\left(\eta_{C}-\xi_{C}\right) N_{C}
$$

$$
N_{C}(t)<\frac{K_{C} N_{C}(0)}{\left(K_{C}-N_{C}(0)\right) \exp \left(-\left(\eta_{C}-\xi_{C}\right) t\right)+N_{C}(0)}<K_{C}
$$

Since $N_{C}(0)<K_{C}$.

Table 1: State variables

\begin{tabular}{|c|l|}
\hline$S_{H}$ & Number of susceptible humans \\
\hline$E_{H}$ & Number of exposed \\
\hline$I_{H}$ & Number of infected humans \\
\hline$E$ & Number of guinea worm eggs \\
\hline$L$ & Number of guinea worm larvae \\
\hline$S_{C}$ & Number of susceptible copepod \\
\hline$I_{C}$ & Number of infected copepod \\
\hline
\end{tabular}


Table 2: List of parameters

\begin{tabular}{|l|l|}
\hline$\eta_{H}$ & Human birth rate $\left(\frac{1}{\text { time }}\right)$ \\
\hline$\xi_{H}$ & Human death rate $\left(\frac{1}{\text { time }}\right)$ \\
\hline$\kappa$ & Recovery rate $\left(\frac{1}{\text { time }}\right)$ \\
\hline$\varepsilon_{H}$ & Human infection fraction $\left(\frac{\text { human infected }}{\text { copepod }}\right)$ \\
\hline$\beta$ & Copepod consumption rate $\left(\frac{\text { copepod consumed }}{\text { human } \times \text { time }}\right)$ \\
\hline$\alpha$ & Worm emergence rate $\left(\frac{1}{\text { time }}\right)$ \\
\hline$\lambda_{0}$ & Visitation rate $\left(\frac{\text { visits to the water }}{\text { time }}\right)$ \\
\hline$\lambda_{1}$ & Egg release rate $\left(\frac{\text { egg released }}{\text { human } \times \text { rate }}\right)$ \\
\hline$\xi_{E}$ & Natural death rate of eggs $\left(\frac{1}{\text { time }}\right)$ \\
\hline$\omega$ & Hatching rate $\left(\frac{1}{\text { time }}\right)$ \\
\hline$\sigma$ & Ratio of matured larvae to the number of eggs hatched $\left(\frac{\text { larvae survived }}{\text { egg }}\right)$ \\
\hline$\xi_{L}$ & Natural death rate of larvae $\left(\frac{1}{\text { time }}\right)$ \\
\hline$\gamma$ & Larvae consumption rate $\left(\frac{\text { larvae consumed }}{\text { copepod } \times \text { time }}\right)$ \\
\hline$\varepsilon_{L}$ & Copepod infection fraction $\left(\frac{\text { copepod infected }}{\text { larvae }}\right)$ \\
\hline$\xi_{C}$ & Natural death rate of copepod $\left(\frac{1}{\text { time }}\right)$ \\
\hline$\Phi_{C}^{\prime}$ & Death rate of infected copepod $\left(\frac{1}{\text { time }}\right)$ \\
\hline & Copepod birth rate $\left(\frac{1}{\text { time }}\right)$ \\
\hline & Larvae saturation constant (larvae $)$ \\
\hline & \\
\hline & \\
\hline & \\
\hline &
\end{tabular}

\section{Equilibrium and Stability Analysis}

In this section, equilibrium and stability analysis of the model are discussed. When modelling infectious diseases, the most important issue that arises is whether the disease spread could attain pandemic level or it could be wiped out. To have a better understanding of the dynamics of the disease, equilibrium and stability analysis is performed.

\subsection{Disease-Free Equilibrium}

For the disease-free equilibrium, we set the disease states and the left-hand side of (2.1)-(2.7) to zero. The resulting system is solved which is given to $\pi_{0}=\left(S_{H}^{*}, 0,0,0,0, S_{C}^{*}, 0\right)$

$$
S_{C}^{*}=K_{C}\left(1-\frac{\beta K_{H}}{\Phi_{C}\left(\eta_{C}-\xi_{C}^{\prime}\right)}\right)<K_{C}
$$

The last inequality holds since $0<\beta K_{H}<\Phi_{C}\left(\eta_{C}-\xi_{C}^{\prime}\right)$

We obtain the reproductive number $R_{0}$ by expressing (2.1) - (2.7) as the difference between the rate of new infection in each infected compartment $F$ and the rate of transfer between each infected compartment $G$.

$$
\left[\begin{array}{c}
\frac{d E_{H}}{d t} \\
\frac{d I_{H}}{d t} \\
\frac{d E}{d t} \\
\frac{d L}{d t} \\
\frac{d I_{C}}{d t}
\end{array}\right]=F-G=\left[\begin{array}{c}
\varepsilon_{C} \beta\left(\frac{I_{C}}{\Phi_{\mathrm{C}}}\right) S_{H} \\
0 \\
\lambda_{0} \lambda_{1} I_{H} \\
0 \\
\varepsilon_{L} \gamma\left(\frac{L}{\Phi_{\mathrm{L}}}\right) S_{C}
\end{array}\right]-\left[\begin{array}{c}
\alpha E_{H}-\xi_{H} E_{H}\left(\frac{E_{H}+I_{H}}{K_{H}}\right) \\
-\alpha E_{H}+\kappa I_{H}+\xi_{H} I_{H}\left(\frac{E_{H}+I_{H}}{K_{H}}\right) \\
\left(\omega+\xi_{E}\right) E \\
-\omega \sigma E+\xi_{L} L+\gamma\left(\frac{L}{\Phi_{\mathrm{L}}}\right) N_{C} \\
\beta\left(\frac{I_{C}}{\Phi_{\mathrm{C}}}\right) N_{H}+\xi_{C}^{\prime} I_{C}\left(1-\frac{N_{C}}{K_{C}}\right)
\end{array}\right]
$$

The Jacobian matrices $J_{F}$ and $J_{G}$ of $F$ and $G$ are found about $\pi_{0}$.

$$
T=J_{F} J_{G}^{-1}=\left[\begin{array}{ccccc}
0 & 0 & 0 & 0 & \frac{\varepsilon_{C} \beta S_{C}^{*} K_{C}}{\beta S_{C}^{*} K_{C}+\xi_{C}^{\prime} \Phi_{C} S_{C}^{*}-\xi_{C}^{\prime} \Phi_{C} S_{C}^{*}} \\
0 & 0 & 0 & 0 & 0 \\
\frac{\lambda_{0} \lambda_{1}}{K} & \frac{\lambda_{0} \lambda_{1}}{K} & 0 & 0 & 0 \\
0 & 0 & 0 & 0 & 0 \\
0 & 0 & \frac{\xi_{L} \gamma S_{C}^{*} \omega \sigma}{\left(\omega+\xi_{E}\right)\left(\xi_{L} \Phi_{L}+\gamma S_{C}\right)} & \frac{\xi_{L} \gamma S_{C}^{*}}{\xi_{L} \Phi_{L}+\gamma S_{C}} & 0
\end{array}\right]
$$


$R_{0}$ is the maximum eigenvalue of $\mathrm{T}$ given as

Clearly, $R_{0} \geq 0$ since $K_{C} \geq S_{C}^{*}$

$$
R_{0}=\sqrt[3]{\frac{\varepsilon_{L} \gamma S_{C}^{*} \omega \sigma \lambda_{0} \lambda_{1} \xi_{C} \beta K_{H} K_{C}}{\kappa\left(\omega+\xi_{E}\right)\left(\xi_{L} \Phi_{L}+\gamma S_{C}^{*}\right)\left(\beta S_{H}^{*} K_{C}+\xi_{C}^{\prime} \Phi_{C} S_{C}^{*}-\xi_{c}^{\prime} \Phi_{C} S_{C}^{*}\right)}}
$$

Theorem 3.1: If one of the diseased classes of an equilibrium point of the system is zero, then all the diseased classes are zero.

Proof: At equilibrium, the left sides of (2-1) - (2.7) are set to zero. Suppose $I_{H}=0$ in (2.4), $\left(\omega+\xi_{E}\right)>$ 0implies $E=0$. In (2.5) we have $\left(\xi_{L}+\frac{\gamma N_{C}}{\Phi_{L}}\right) L=0$, but $\left(\xi_{L}+\frac{\gamma N_{C}}{\Phi_{L}}\right)>0$ by our assumption, therefore $L=0$. It follows from (2.7) that $I_{C}=0$ since $\beta, I_{C}, \Phi_{\mathrm{C}}, N_{H}, \xi_{c}^{\prime}, N_{C}$ and $K_{C}$ are positive. Finally, we have from (2.2) that $E_{H}=0$ if $I_{C}=0$.

Table 3: Parameter values for the disease-free area (Ghana) and endemic area (Ethiopia) with time in days

\begin{tabular}{|c|l|l|l|l|l|l|l|}
\hline Parameter & $\begin{array}{l}\text { Diseases } \\
\text { free area }\end{array}$ & $\begin{array}{l}\text { Endemic } \\
\text { area }\end{array}$ & Reference & Parameter & $\begin{array}{l}\text { Diseases } \\
\text { free area }\end{array}$ & $\begin{array}{l}\text { Endemic } \\
\text { area }\end{array}$ & Reference \\
\hline$\eta_{\mathrm{H}}$ & $\begin{array}{l}8.767 \\
\times 10^{-5}\end{array}$ & $1.055 \times 10^{-1}$ & {$[10]$} & $\xi_{\mathrm{H}}$ & $\begin{array}{l}2.11 \times 10^{-} \\
5\end{array}$ & $2.548 \times 10^{-5}$ & {$[10]$} \\
\hline$\kappa$ & 0.0222 & 0.0222 & calculated & $\varepsilon_{\mathrm{C}}$ & 0.004 & 0.024 & calculated \\
\hline$\beta$ & 0.5 & 2 & Calculate & $\alpha$ & 0.00274 & 0.00274 & calculated \\
\hline$\lambda_{0}$ & 0.002 & 0.02 & Assumed & $\lambda_{1}$ & 3000000 & 3000000 & {$[2]$} \\
\hline$\xi_{\mathrm{E}}$ & 0.333 & 0.333 & {$[2]$} & $\omega$ & 0.072 & 0.072 & {$[9]$} \\
\hline$\sigma$ & 0.4 & 0.4 & Calculate & $\xi_{\mathrm{L}}$ & 0.0333 & 0.0333 & {$[3]$} \\
\hline$\gamma$ & 3 & 3 & {$[3]$} & $\varepsilon_{\mathrm{L}}$ & 0.1 & 0.1 & assumed \\
\hline$\xi_{\mathrm{C}}$ & 0.005 & 0.005 & {$[2]$} & $\eta_{\mathrm{C}}$ & 0.75 & 0.75 & {$[2]$} \\
\hline$\xi_{\mathrm{C}}$ & 0.125 & 0.125 & {$[3]$} & $\Phi_{\mathrm{C}}$ & 200000 & 200000 & \\
\hline $\mathrm{K}_{\mathrm{H}}$ & 1000 & 1000 & assumed & $\mathrm{K}_{\mathrm{C}}$ & 100000 & 100000 & assumed \\
\hline$\Phi_{\mathrm{L}}$ & 500000 & 5000000 & & & & & \\
\hline
\end{tabular}

\subsection{Bifurcation}

We consider two parameters $\lambda_{0}$ and $\xi_{\mathrm{L}}$ as means of intervention. Education is the intervention represented by $\lambda_{0}$ and continuous water treatment with the use of larvicide by $\xi_{\mathrm{L}}$. By reducing the visitation rate, our model reveals that $\pi_{0}$ is stable if $0<\lambda_{0}<0.00115$.

Water treatment with the use of larvicide increases the death rate of guinea worm larva. Our model describes that $\pi_{0}$ is stable if $\xi_{\mathrm{L}}>0.1016$.

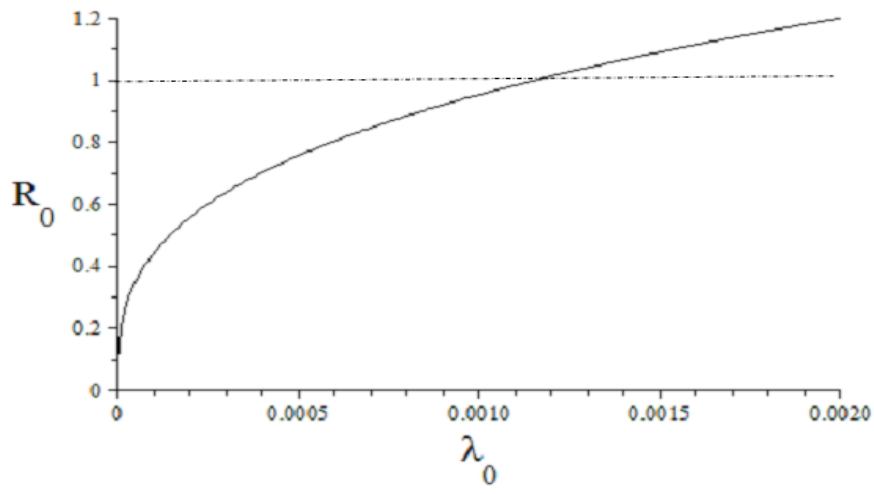

Figure 1: Intervention parameter $\lambda_{0}$ (education) 


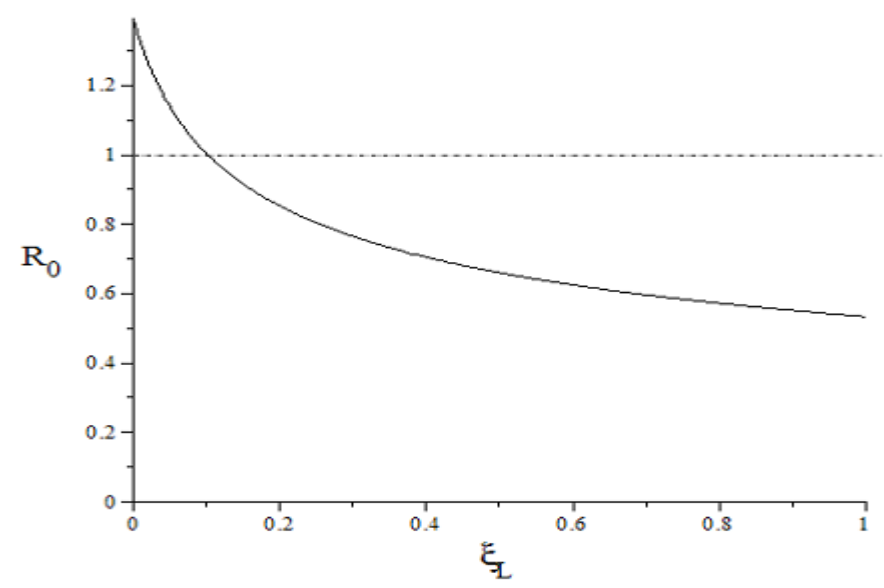

Figure 2: Intervention parameter $\xi_{L}$ (water treatment)

\subsection{Endemic Equilibrium}

Theorem 3.2:If $\lambda_{0} \lambda_{1} I_{H}<\left(\omega+\xi_{E}\right) E, \quad t \geq 0$, there exist three equilibria

$$
\begin{gathered}
\pi_{1}=\left(\mathrm{K}_{\mathrm{H}}, 0,0,0,0,0,0\right) \\
\pi_{2}=\left(0,0,0,0,0, \mathrm{~K}_{\mathrm{C}}, 0\right) \text { and } \\
\pi_{3}=\left(K_{H}, 0,0,0,0, S_{C}^{*}, 0\right)
\end{gathered}
$$

where $S_{C}^{*}=K_{C}\left(1-\frac{\beta K_{H}}{\Phi_{C}\left(\eta_{C}-\xi_{C}^{\prime}\right)}\right)$. $\pi_{1}$ is unstable while $\pi_{2}$ and $\pi_{3}$ are locally stable.

Proof: Set the left side of (2.1)-(2.7) to zero. Putting $\lambda_{0} \lambda_{1} I_{H}=c\left(\omega+\xi_{E}\right) E, 0 \leq c<1$, fort $\geq 0$, in (2.4), we have $E=0$. This implies, by Lemma 2.1 and Theorem 3.1, that $E_{H}=I_{H}=L=I_{C}=0$, thus, (2.1) - (2.7) is reduced to

$$
\begin{aligned}
& 0=\left(\eta_{H}-\xi_{H}\right) S_{H}\left(1-\frac{S_{H}}{K_{H}}\right) \\
& 0=\left(\eta_{C}-\xi_{C}\right) S_{C}\left(1-\frac{S_{C}}{K_{C}}\right)-\beta\left(\frac{S_{C}}{\Phi_{C}}\right) S_{H} 3.2
\end{aligned}
$$

Neglecting the trivial case, $\pi_{1}, \pi_{2}$ and $\pi_{3}$ are obtained from (3.1) and (3.2).

We check the stabilities of $\pi_{1}$ and $\pi_{3}$ (because $\pi_{3}=\pi_{2}$ when $K_{H}=0$ ) by finding the eigenvalues of the Jacobian matrices of system (2.1)-(2.7) evaluated at $\pi_{1}$ and $\pi_{2}$ respectively [11,12].

To check the stability of $\pi_{1}$, we have the Jacobian matrix

where

$$
J_{\pi_{1}}=\left[\begin{array}{ccccccc}
-\eta_{H}+\xi_{H} & -\eta_{H}+\xi_{H} & \Gamma & 0 & 0 & 0 & -\frac{\mathcal{E}_{C} \beta K_{H}}{\Phi_{C}} \\
0 & -\alpha & 0 & 0 & 0 & 0 & \frac{\varepsilon_{C} \beta K_{H}}{\Phi_{C}} \\
0 & \alpha & -\kappa & 0 & 0 & 0 & 0 \\
0 & 0 & 0 & \beth & 0 & 0 & 0 \\
0 & 0 & 0 & \omega \sigma & -\xi_{L} & 0 & 0 \\
0 & 0 & 0 & 0 & 0 & T & \eta_{C} \\
0 & 0 & 0 & 0 & 0 & 0 & -\frac{\beta K_{H}}{\Phi_{C}}-\xi_{C}^{\prime}
\end{array}\right]
$$

$$
\begin{aligned}
& \Gamma=-\eta_{H}+\xi_{H}+\kappa \\
& \beth=(c-1)\left(\omega+\xi_{E}\right) \\
& \nearrow=\eta_{C}-\frac{\beta K_{H}}{\Phi_{C}}-\xi_{C}
\end{aligned}
$$

and the eigenvalues are

$$
y_{1}=\left(\begin{array}{c}
-\kappa \\
-\alpha \\
-\frac{\beta K_{H}}{\Phi_{C}}-\xi_{C}^{\prime} \\
\eta_{C}-\frac{\beta K_{H}}{\Phi_{C}}-\xi_{C} \\
-\xi_{L} \\
(c-1)\left(\omega+\xi_{E}\right) \\
-\eta_{H}+\xi_{H}
\end{array}\right)
$$


$\pi_{1}$ is not stable since $\eta_{C}-\frac{\beta K_{H}}{\Phi_{C}}-\xi_{C}>0$

For $\pi_{3}$, we have the Jacobian matrix

where

$$
J_{\pi_{3}}=\left[\begin{array}{ccccccc}
-\eta_{H}+\xi_{H} & -\eta_{H}+\xi_{H} & \Gamma & 0 & 0 & 0 & -\frac{\varepsilon_{C} \beta K_{H}}{\Phi_{C}} \\
0 & -\alpha & 0 & 0 & 0 & 0 & \frac{\varepsilon_{C} \beta K_{H}}{\Phi_{C}} \\
0 & \alpha & -\kappa & 0 & 0 & 0 & 0 \\
0 & 0 & 0 & \beth & 0 & 0 & 0 \\
0 & 0 & 0 & \omega \sigma & -\xi_{L}-\frac{\gamma s_{C}^{*}}{\Phi_{L}} & 0 & 0 \\
-\frac{\beta s_{C}^{*}}{\Phi_{C}} & -\frac{\beta S_{C}^{*}}{\Phi_{C}} & -\frac{\beta s_{C}^{*}}{\Phi_{C}} & 0 & -\frac{\varepsilon_{L} \gamma s_{C}^{*}}{\Phi_{L}} & \Theta & \Psi \\
0 & 0 & 0 & 0 & \frac{\varepsilon_{L} \gamma s_{C}^{*}}{\Phi_{L}} & 0 & \Xi
\end{array}\right]
$$

$\Theta=\left(\eta_{C}-\xi_{C}\right)\left(1-\frac{2 S_{C}^{*}}{K_{C}}\right)-\frac{\beta K_{H}}{\Phi_{C}}$

$\Psi=\eta_{C}-\frac{\left(2 \eta_{C}-\xi_{C}\right) S_{C}^{*}}{K_{C}}$

$\Xi=-\frac{\beta K_{H}}{\Phi_{C}}-\xi_{C}^{\prime}\left(1-\frac{S_{C}^{*}}{K_{C}}\right)$

and the eigenvalues are

Therefore, $\pi_{3}$ is locally stable when $c<1$.

$$
y_{2}=\left(\begin{array}{c}
-\kappa \\
-\alpha \\
-\left(\eta_{C}-\xi_{C}\right)+\frac{\beta K_{H}}{\Phi_{C}} \\
-\frac{\beta K_{H}\left(\eta_{C}-\xi_{C}+\xi_{C}^{\prime}\right)}{\Phi_{C}\left(\eta_{C}-\xi_{C}\right)} \\
(c-1)\left(\omega+\xi_{E}\right) \\
-\eta_{H}+\xi_{H} \\
-\xi_{L}-\frac{\gamma K_{C}\left(\Phi_{C}\left(\eta_{C}-\xi_{C}\right)-\beta K_{H}\right)}{\Phi_{L} \Phi_{C}\left(\eta_{C}-\xi_{C}\right)}
\end{array}\right)
$$
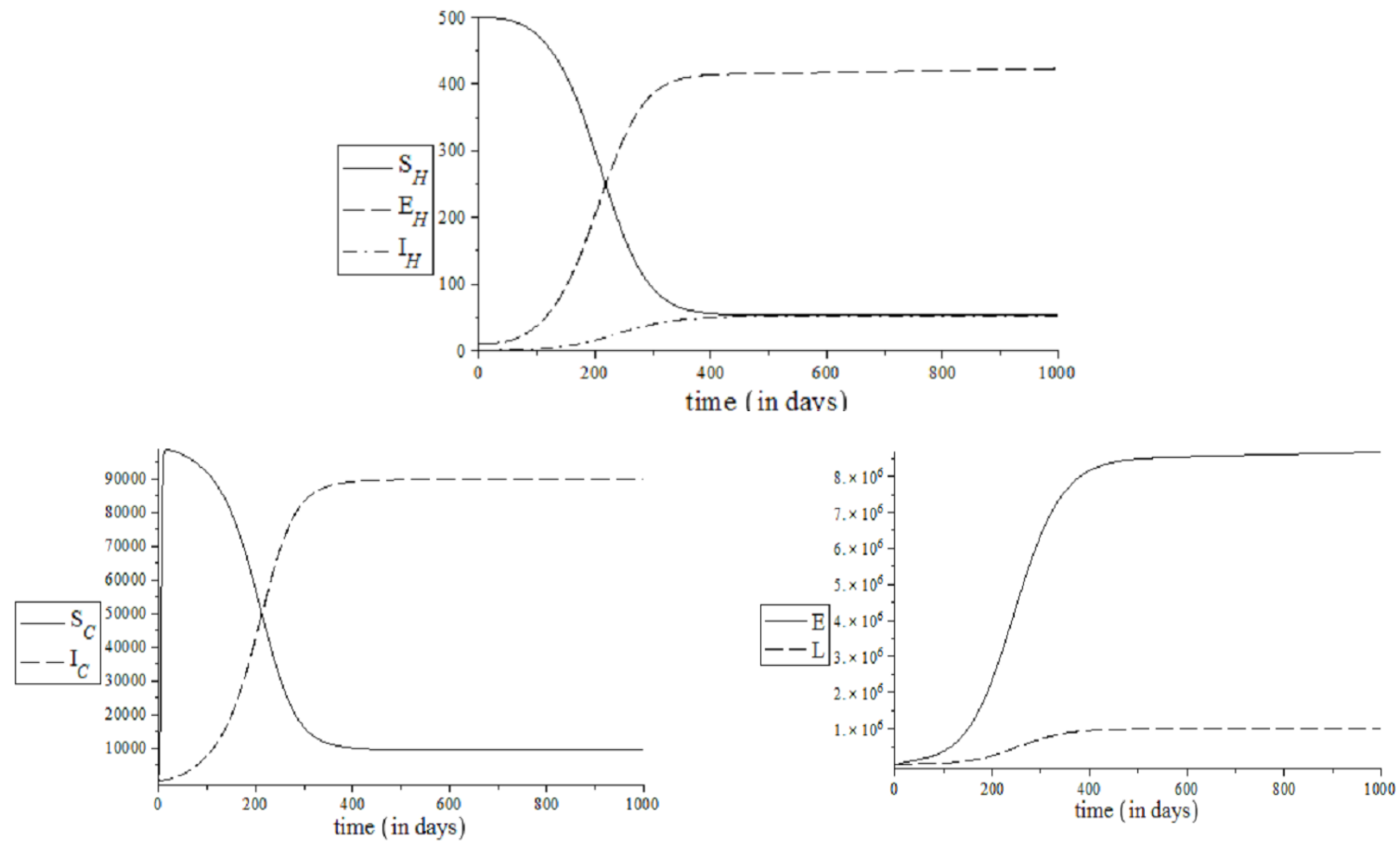

Figure 3: $S_{H}(0)=500, E_{H}(0)=10, I_{H}(0)=500, E(0)=10000, L(0)=5000, S_{C}(0)=1000, I_{C}(0)=500$ 
We stimulated the endemic equilibrium using the parameter values in Table 3. The results show that the disease is present in all the population classes.

To check Theorem 3.2, we stimulated the endemic equilibrium with $\lambda_{0}=0$. The results agree with Theorem 3.2. This means that if the education about the disease is effective and the visitation rate is reduced, the disease will be wiped out.

Using the data in Table 3, we stimulated the case when the intervention parameter $\xi_{\mathrm{L}}$ (i.e. water treatment) is effective. It was observed that if the water treatment is effective to the level that the life span of larvae is reduced to 147 minutes (i.e. $\xi_{\mathrm{L}}=9.796$ per day), the population of guinea worm egg remains in the system but other disease classes decrease as time increases.

\section{Conclusion}

We have highlighted a compartmental modelling approach for three different populations-human, vector and parasite. It is assumed that the human and vector populations grow logistically and parasite population increases by infected human's visitation to the river. The model is analysed for disease-free and endemic equilibria. Local stability of the non-trivialequilibria in both cases is guaranteed only under certain conditions.

For disease-free equilibrium, keeping the intervention parameter values in the value intervals given in section 3.2 allows the system to remain disease-free, the endemic equilibria $\pi_{2}$ and $\pi_{3}$ are stable when $\lambda_{0} \lambda_{1} I_{H}(t) \leq\left(\omega+\xi_{E}\right) E(t)$. It is concluded from the analysis and simulation that if the intervention parameter (visitation rate) $\lambda_{0} \leq \frac{\left(\omega+\xi_{E}\right) E}{\lambda_{1} I_{H}}\left(I_{H} \neq 0\right)$, the spread of disease decreases and could be wiped out. By computer simulation it is shown that if the larvicide is effective (i.e. $\xi_{\mathrm{L}}$ is increased) to the point when $\frac{d L}{d t} \leq 0$, then the endemic equilibrium is locally stable.
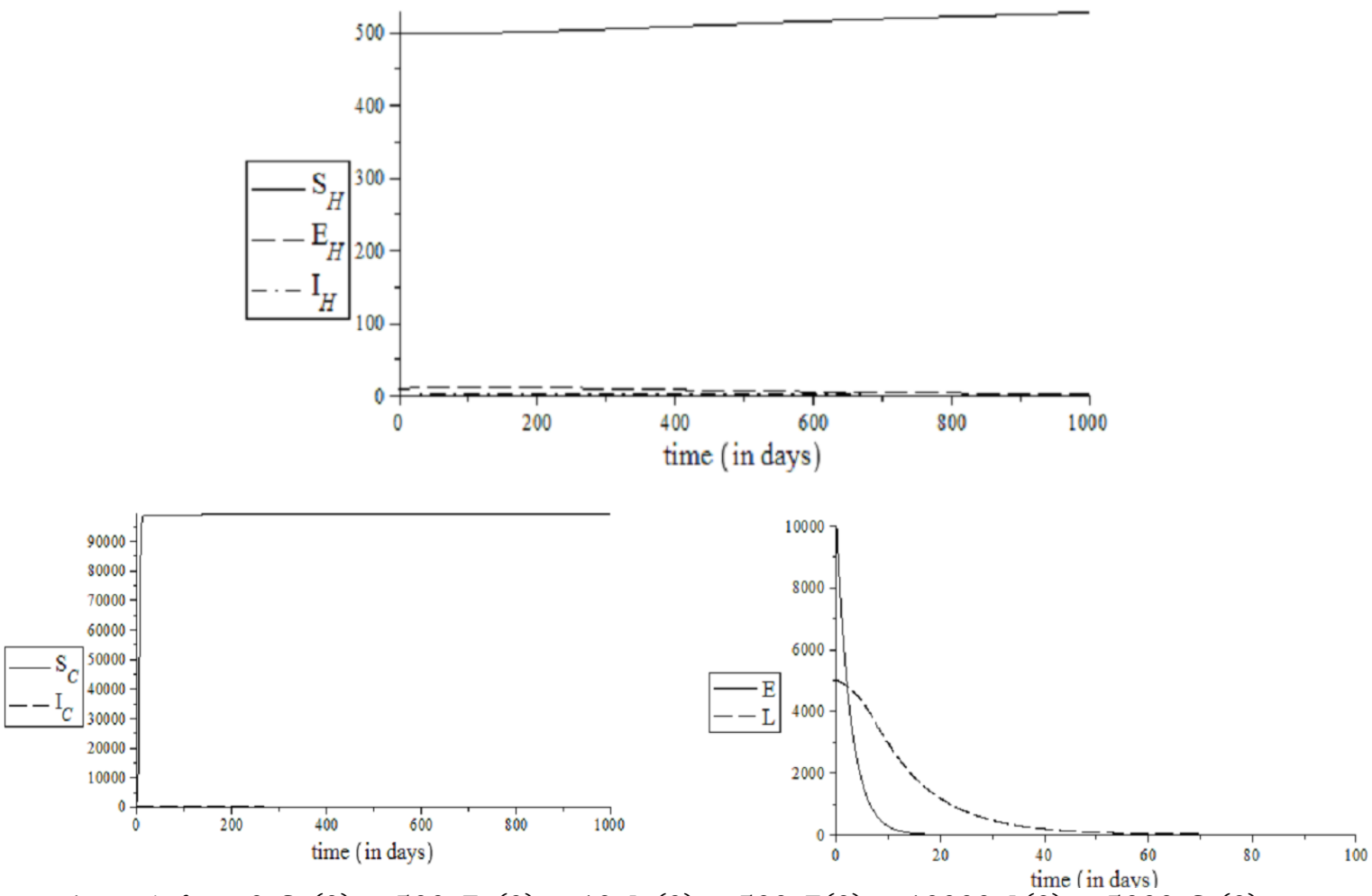

Figure 4: $\lambda_{0}=0, S_{H}(0)=500, E_{H}(0)=10, I_{H}(0)=500, E(0)=10000, L(0)=5000, S_{C}(0)=$ $1000, I_{C}(0)=500$

It is also found by simulation that reducing the parasite birth rate (which can be achieved by educating people not to put infected limbs into the drinking water) is more effective than water treatment. This model can be a useful tool in the control of the spread of dracunculiasis and the understanding of the model formulation can help in modelling any other water-borne disease. 


\section{References}

[1] Anosike, J.C.; Azoro, VA; Nwoke, B.E.B; Keke, IR; Okere, AN; Oku, EE; Ogbul JN; Tony-Njoku, F: Okoro, O.U., Nwosu, D.c.Dracunculiasis in the North Eastern Border of Ebonyi State, South Eastern Nigeria. Int. J. Egg. Environm. Hith, 205,2002 , 1-7.

[2] Cyclops an intermediate host for guinea worm. http://www.who.int/water sanitation health/resources/vector324 to 336.pdf Accessed May 25, 2013-09-28

[3] SarojBapnaRelative Susceptibilities of Cyclops species from Rajasthan State of Guinea Worm (Dracunculiasismedinansis) larvae. Bulletin of the World Health Organisation, 63(5), 1985, 881-886.

[4] St. George, J. Bleeding in Pregnancdy due to re-troplacental situation of guinea worm. Annals of Tropical Medicine and Parasitology 69, 1975, 383-386.

[5] Robert J.S., Patrick C., James H., Alex D. A Mathematical Model for the eradication of guinea worm disease. Understanding the dynamics of emerging and re-emerging infectious diseases using mathematical models pgs. 2012, 133-156.

[6] Ruiz-Tiben E., Hopkins D.R.Dracunculiasis (Guinea Worm Disease) eradication. Adv. Parasitol 61, 2006, 275-309

[7] World Health Organisation. World Health Assembly, Resolution WHA 57.9. Elimination of dracunculiasis: resolution of the 57 World Health Assembly, Geneva, Switzerland: http//www.whmv/gb/ebwha/pdf_files/wha57/a57_r9-en.pdf Accessed April 25 2013.

[8] Adetunde I.A. The Epidemiology of guinea worm infection in Tamale District, in the Northern Region of Ghana. Journal of Modern Mathematics and Statistics. 2008, 50-54

[9] Kathryh L. Guinea worm disease (Dracunculiasis): Opening a mathematical can of worms. M.Sc. Thesis, Bryn Mawr College, Pennsylvania, USA, 2012.

[10] CIA World Factbook (2012). http://www.cia.gov/library/publications/the-wrold-factbook/rankorder/2054rank.html

[11] Blanchard p., Daveney R.L., Hall G.R.; (2006). Differential Equations (Thomson Brooks/Cole, Belmont, C.A. 2006), 323

[12] Hirsch, M.W., Smale S. Differential Equations. Dynamical Systems and Linear Algebra. (Academic Press Inc. Harcourt Brace Javanovich Publishers, 1974) 185-191 